\title{
Balkanologie
}

Balkanologie Revue d'études pluridisciplinaires

Vol. VII, n 2 | 2003

Volume VII Numéro 2

\section{Partis politiques et électorats paysans en Bulgarie et en Roumanie}

Antoine Roger

\section{OpenEdition}

12 Journals

Édition électronique

URL : http://journals.openedition.org/balkanologie/502

DOI : $10.4000 /$ balkanologie. 502

ISSN : 1965-0582

Éditeur

Association française d'études sur les Balkans (Afebalk)

Édition imprimée

Date de publication : 1 décembre 2003

Pagination : 151-179

ISSN : 1279-7952

\section{Référence électronique}

Antoine Roger, «Partis politiques et électorats paysans en Bulgarie et en Roumanie », Balkanologie [En ligne], Vol. VII, n² 2 | 2003, mis en ligne le 19 février 2009, consulté le 17 décembre 2020. URL : http:// journals.openedition.org/balkanologie/502 ; DOI : https://doi.org/10.4000/balkanologie.502

\section{(c) Tous droits réservés}




\title{
PARTIS POLITIOUES ET ÉLECTORATS PAYSANS EN BULGARIE ET EN ROUMANIE
}

\author{
Antoine Roger*
}

Après l'effondrement du bloc soviétique, la vie politique a connu des évolutions similaires en Bulgarie et en Roumanie. Dans les deux pays, une formation dirigée par d'anciens communistes reconvertis est parvenue à s'imposer : le Parti socialiste bulgare (Balgarska Socialisticeska Partija, BSP) a exercé le pouvoir en 1991 puis entre 1992 et 1997; après avoir gouverné la Roumanie entre 1991 et 1996, le parti de Ion Iliescu est revenu aux affaires en novembre $2000^{1}$. L'une et l'autre formation ont trouvé leurs principaux soutiens dans l'électorat paysan ${ }^{2}$. Les élections municipales leur ont permis d'obtenir des mandats exécutifs dans la grande majorité des communes rurales?.

Pour expliquer cet état de fait, deux thèses ont été avancées que nous désignerons respectivement thèse de la désagrégation et thèse de l'imprégnation.

La thèse de la désagrégation met l'accent sur la culture politique des pays

\footnotetext{
- Institut d'Etudes Politiques de Bordeaux.

${ }^{1}$ Le Front de salut national (FrontuI Salvarii Nationale - FSN) a formè un premier gouvemement en mai 1990. Il s'agissait toutefois d'une formation mal cristallisée, abritant à la fois les proches du Premier ministre Petre Roman et les partisans de Ion Iliescu. La crise gouvernementale de septembre 1991 a permis à ces derniers de faire scission et de constituer le Front démocratrique de salut national (Frontul Democratic Salvarii Nationale - FDSN) pour exercer seuls le pouvoir. Cette formation a été rebaptisée Parti de la democratie sociale de Roumanie (Partidul Democratiei Sociale din Románia - PDSR) en juin 1993. Elle a conservé cette désignation jusqu'en juin 2001. En fusionnant avec le microscopique Parti social démocrate roumain (Partidul Social Democrat Român - PSDR), elle s'est alors transformée en Parti social démocrate (Partidul Sacial Democrat).

${ }^{2}$ La population rurale représente $29,3 \%$ de la population totale en Bulgarie selon le recensement de 1992 et $52,9 \%$ en Roumanie selon une enquēte démographique réalisée en 1995 .

3 Cf. Shopov (Vladimir), "How the Voters respond in Bulgaria ", in Lawnson (Kay), Rommele (Andrea), Karasimeonov (Georgi), eds., Cleavages, Parties and Voters. Studies from Bulgaria, the Czech Republic, Hungary, Poland and Romania, London : Routledge, 1999, p. 192 ; Berindei (Minhea), " Résultats des élections locales roumaines de juin 1996 ", La Nouvelle Alternative, (44), 1996.
} 
considérés. Selon ses défenseurs, les autorités communistes ont détruit la société civile locale ; pour prévenir la formation de tout système de loyauté concurrent, elles ont éliminé les mécanismes solidarité traditionnels qui s'étaient formés au sein des communautés villageoises 4 . Après le changement de régime, les campagnes se trouvent par conséquent dépourvues de toute organisation agrégative susceptible de donner forme à un cadre d'opinion structuré; en résulte une personnalisation de la vie politique locale ${ }^{5}$. Cette tendance est confortée par le personnel politique lui-même : les représentants locaux des partis s'attachent à construire une clientèle plutôt qu'à animer le débat public ; leurs programmes électoraux ne sont guère tranchés et l'étiquette politique dont ils s'affublent ne remplit qu'une fonction ornementale. Rompus aux techniques de propagande et habiles à manipuler un électorat faiblement informé, les dirigeants locaux qui exerçaient quelque responsabilité sous le régime communiste ont sur leurs concurrents un net avantage ${ }^{6}$. L'analyse ainsi proposée repose sur des bases fragiles : des recherches poussées ont montré l'existence de conflits d'intérêts locaux sous le régime communiste ; ces conflits n'entrent pas dans les cadres de référence occidentaux ; ils n'en constituent pas moins des facteurs de structuration significatifs?

La thèse de l'imprégnation invite précisément à examiner les structures politiques instituées. Selon ses concepteurs, le régime communiste a favorisé la formation de baronnies locales. Les édiles municipaux ont usé de leur influence au sein du Parti pour obtenir que leurs administrés bénéficient d'avantages ponctuels ou de dérogations. Cette pratique était particulièrement répandue dans les zones rurales : le chaos administratif qui régnait dans la plu-

4 Cf. Baldersheim (Harald), mlner (Michael), "Local Democracy : The Challenges of InstitutionBuilding ", in Baldersheim (Harald), et. al., Local democracy and the processes of transformation in EastCentral Europe, Boulder : Westview, 1996, pp. 4, 11, 15.

5 Cf. Offerdal (Audun), Hanspach (Dan), Kowalczyk (Andrzej), Patocka (Jiri), " Elites and Parties : the New Local Elites ", in Baldersheim (Harald), et. al., op. cit., pp. 109, 137.

${ }^{6}$ Cf. Regulska (Joanna), "Local government reform in central and eastern Europe ", in Bennett (John), ed., Local government in the new Europe, London : Belhaven Press, 1993, pp. 184-185; Gibson (John), Hanson (Philip), " Decentralization and Change in Post-Communist Countries ", in Gibson (John), Hanson (Philip), eds., Transformation from below : local power and the political economy of post-communist transitions, Cheltenham : Edward Elgar, 1996, p. 309 ; Rose (Lawrence), Buchta (Stanislav), Gadjuschek (György), Grochowski (Miroslav), Hubacek (Ondrej), " Political Culture and Citizen Involvement ", in Baldersheim (Harald), et. al., op. cit., pp. 98-99 ; Szakolczai (Arpad), Types of Mayors, Types of Subjectivity : Continuities and Discontinuities in the East-Central European Transitions, European University Institute [Working paper SPS (93/5)], 1993, pp. 34-39 ; Szakolczai (Arpad), Re-Building the Polity : A Comparative Study of Mayors in the Hungarian, Czech and Slovakian Republics, European University Institute [Working paper SPS (93/8)], 1993, pp. 11-12, 17.

7 Cf. Verdery (Katherine), Transylvanian Villagers : Three Centuries of Political Economic and Ethnic Change, Berkeley : University of California Press, 1983, pp. 37, 56 ; Kideckel (David A.), The Solitude of Collectivism : Romanian Villager to the Revolution and Beyond, Ithaca, Cornell University Press, 1993, pp. 138-148 ; Creed (Gerald W.), Domesticating Revolution. From Socialist Reform to Ambivalent Transition in a Bulgarian Village, University Park : The Pennsylvania State University Press, 1998, pp. 205-218. 
part des villages a permis d'atteindre un degré d'indépendance locale sans équivalent et de développer des systèmes de patronage particulièrement efficaces. Ces systèmes sont demeurés en place après l'effondrement du régime communiste. Les anciennes élites locales sont parvenues à protéger leur source de pouvoir ; sitôt accoutumées au nouveau contexte institutionnel, elles se sont réclamées des formations dirigées par des cadres communistes reconvertis et ont recommencé à négocier avec les autoritès centrales pour distribuer des ressources et des faveurs aux paysans ${ }^{8}$. Contrairement à la précédente, cette explication prend en compte les particularités du régime communiste. Elle tend cependant à les réifier : étant admis que des réseaux clientélistes ont été construits dans le passé 9 , ils ne sauraient fonctionner sur un mode identique dans la période présente ; l'instabilité générée par les réformes économiques nuit à leur bon fonctionnement et compromet leur survie ${ }^{10}$.

Si les thèses de la désagrégation et de l'imprégnation adoptent des orientations symétriques, elles présentent somme toute un même travers : les variables politiques héritées de la période communiste y sont présentées comme des scories qui empêchent d'accorder la vie politique locale au système de partis institué à l'échelle nationale. La conscience politique de l'électorat rural ayant été étouffée ou les héritiers de l'ancien système faisant obstacle à l'émergence de nouvelles élites municipales, les formations constituées ne pour-

\footnotetext{
${ }^{8}$ Coulson (Andrew), "From Democratic Centralism to Local Democracy ", in Coulson (Andrew), ed., Local government in Eastern Europe : establishing democracy at the grassroots, Aldershot : Edward Elgar, 1994, p. 9. Voir aussi : Meurs (Mieke), Begg (Robert), “ Path dependence in Bulgarian Agriculture ", in Pickles (John), Smith (Adrian), eds., Theorising Transition : The Political Economy of Post-communist Transformations, London: Routledge, 1998, pp. 247, 253 ; Kideckel (David A.), "Two Incidents on the Plains in Southern Transylvania : Pitfalls of Privatization in a Romanian Community ", in Kideckel (David A.), ed., East European Communities : The Struggle for Balance in Turbulent Times, Boulder : Westview Press, 1995, p. 51.

${ }^{9}$ Le fonctionnement de ces réseaux est lui-même sujet à discussion. Des recherches ont montré que les autorités communistes s'attachaient à entretenir la confusion entre intégration verticale et intégration horizontale. L'intégration verticale désigne le " processus par lequel les acteurs politiques locaux cherchent a mobiliser et à organiser les ressources locales en vue d'atteindre les objectifs politiques centraux ". L'intégration horizontale caractérise la capacité des responsables locaux à influer sur les décisions des organes centraux pour faciliter la poursuite d'intérêts locaux (cf. Nelson (Daniel N.), « Vertical Intergration and Political Control in Eastem Europe : The Polish and Romanian Cases ", Slavic Review, 40 (2), 1981, pp. 210-213). En introduisant des mesures de décentralisation factices, les dirigeants communistes sont parvenus à renforcer l'intégration verticale tout en laissant croire à un progrès de l'intégration horizontale (cf. Nelson (Daniel N.), "Sub-National Political Elites in a Communist System ", East European Quarterly, to (4), 1976, pp. 459-494; Nelson (Daniel N.), « Background Characteristics of Local Communist Elites: Change Versus Continuity in the Romanian Case ", Polity, 10 (3), 1978, pp. 398-415; Nelson (Daniel N.), Democratic Centralism in Romania : A Study of Local Communist Politics, Boulder : Columbia University Press, 2980 ; Nelson (Daniel N.), Elite-Mass Relations in Communist Systems, Basingstoke : MacMílan, 1988).
}

${ }^{10}$ Prickrance (Chris), “ Mediating Institutions in the Transition from State Socialism : The Case of Local Governement n, in Grabher (Gemot), Stark (David), eds., Restructuring networks in post-socialism : legacies, linkages, and localities, Oxford : Oxford University Press, 1997, pp. 315-318. 
raient s'implanter significativement dans les espaces politiques locaux. Si le BSP et les partisans de Ion Iliescu recueillent la majorité des suffrages dans les campagnes, ce résultat ne serait aucunement le fait d'une implantation locale supérieure à celle des formations concurrentes. Il révèlerait bien plutôt un défaut généralisé d'implantation. Dès lors que cette optique est retenue, les similitudes observées entre la Bulgarie et la Roumanie peuvent être expliquées de la plus simple façon : des scories de même nature sont constituées dans l'un et l'autre cas ; tout au plus est-il utile de marquer quelques variations volumétriques et d'opérer un classement selon que les liaisons entre les scènes politiques nationale et locales sont plus ou moins obstruées.

En redéfinissant les postulats de l'analyse, nous pouvons proposer une méthode de comparaison alternative. Plutôt qu'une scorie dont il faudrait attendre l'éventuelle érosion, l'héritage communiste est selon notre lecture un élément de structuration dynamique. Loin de se perpétuer sous une forme inaltérée, il participe d'un "fondu enchaîné" sociologique : il importe d'étudier les modalités selon lesquelles il se combine avec des principes d'organisation nouveaux pour donner forme à des arrangements sociaux inédits. Les rapports qui se sont établis entre les campagnes et le pouvoir communiste ne sont pas reproduits à l'identique dans la période présente ; ils forment tout au plus une matrice. Aussi des comparaisons qualitatives sont-elles nécessaires : plutôt que de chercher des structures communes dans les campagnes bulgares et roumaines, il faut considérer que des logiques différentes peuvent produire des effets similaires ; plutôt que de marquer des différences de degré entre les exemples considérés, il convient de caractériser plusieurs "fondus enchaînés".

Plusieurs niveaux d'observation doivent être croisés ${ }^{11}$. Les "fondus enchaînés" entre dispositifs hérités du régime communiste et nouveaux facteurs de structuration sont observés au niveau national aussi bien qu'au niveau local : " les changements à l'œuvre dans les macro-institutions peuvent affecter le fonctionnement des micro-institutions "; les tensions observées au niveau micro-institutionnel génèrent dans le même temps des effets propres et contribuent à l'émergence de nouvelles macro-institutions ${ }^{12}$.

Appliqué aux partis politiques bulgares et roumains, ce schéma d'analyse invite à caractériser un rapport dynamique entre les programmes agraires définis au niveau national et la structuration des espaces politiques locaux. Les formations qui s'attirent les faveurs des paysans ne sont pas les bénéficiaires indirectes d'une incapacité générale à articuler les divisions politiques natio-

${ }^{11} \mathrm{Cf}$. Sawicki (Frédéric), " Les politistes et le microscope ", in CURAPP, Les méthodes au concret, Paris : PUF, 2000.

12 Cf. Burawoy (Michael), Verdery (Katherine), "Introduction ", in Burawoy (Michael), Verdery (Katherine), eds., Uncertain Transition : Ethnographies of Change in Postsocialist World, Lanham : Rowman \& Littlefield, 1999, pp. 2, 7, 15. 
nales et les affrontements locaux. Elles s'imposent pour la raison qu'elles parviennent à combiner plus efficacement que leurs concurrentes les niveaux macro-institutionnel et micro-institutionnel.

Les programmes agraires dont se dotent les partis politiques relèvent du niveau macro-institutionnel. Ils sont modelés par les recommandations économiques que formule l'Union européenne dans le cadre du processus d'élargissement. La Commission réclame une décollectivisation complète de la terre, une libéralisation des prix et une privatisation du système de distribution. Elle entend que des grandes exploitations soient constituées et orientées, sur le modèle des fermes occidentales, vers une agriculture intensive. Les fermes collectives doivent être démantelées et céder la place à des grandes exploitations individuelles ; ces principes une fois observés, l'effectif de la paysannerie sera considérablement réduit : l'industrie privée fournira de nouveaux emplois et permettra de prompts recyclages individuels ${ }^{13}$. Les partis politiques bulgare et roumain doivent se positionner face aux mots d'ordre ainsi énoncés. Ils mettent au point trois types de programmes agraires que nous désignons respectivement anti-intégrationniste, intégrationniste et conciliatoire ${ }^{14}$.

Le programme anti-intégrationniste propose de suivre une voie originale, adaptée aux ressources locales et aux structures héritées de la période communiste. Selon ses promoteurs, il convient de renoncer à tout dialogue avec l'Union européenne pour maintenir ou ramener l'économie nationale dans des formes d'organisation collectives et centralisées. Les paysans sont appelés à se rassembler dans des fermes d'État. Ces propositions sont défendues par le Parti communiste bulgare (Balgarska Komunistices ka Partija, BKP) et par son homologue roumain le Parti socialiste du travail (Partidul Socialist al Muncitorii, PSM). Elles sont également le fait de factions constituées au sein du BSP et du parti formé autour de Ion Iliescu ${ }^{15}$.

\footnotetext{
${ }^{13} \mathrm{Cf}$. Commission Européenne, "Agricultural Situation and Prospects in the Central and Eastern Europe Countries : Bulgaria n, Directorate General for Agriculture (DG VI) Working Document, 1998 ; Commission Européenne, "Agricultural Situation and Prospects in the Central and Eastern Europe Countries : Romania ", Directorate General for Agriculture (DG VI) Working Document, 1998 ; Lhomel (Edith), " Le dossier agricole dans les négociations d'adhésion à l'Union européenne : une question cruciale ", La Nouvelle Alternative, (3), 1999 ; Ezkenazi (Ilko), Nikolov (Krasimir), " Relations with the European Union : Developments to Date and Prospects ", in Zloch-Christy (lliana), ed., Bulgaria in Time of Change : Economic and Political Dimensions, Aldershot : Avebury, 1996, pp. 195-197 ; Rauta (Corneliu), " L'impact de l'adhésion à l'Union Européenne sur l'agriculture de la Roumanie ", Romanian Journal of International Affairs, 2 (4), 1996, pp. 135-136, 141.
}

\footnotetext{
${ }^{14}$ Roger (Antoine), " The Influence of the European Union on Political Orientations of Ethnic Minorities : Comparing Post-communist Bulgaria, Romania and Latvia n, communication au colloque Voice or Exit : Comparative Perspectives on Ethnic Minorities in Twentieth Century Europe, Berlin : Humboldt Universität, 17-18 juin 2001.

15 Cf. Wallex (Michael), "Making and Breaking : Factions in the Process of Party Formation in Bulgaria ", in Gillespie (Richard), Waller (Michael), Lopez Nieto (Lourdes), eds., Factional Politics and democratization, London : Frank Cass, 1995 ; Serb (Sorin), « PDSR n, Sfera politicii, (18), 1994
} 
Le programme intégrationniste invite à une stricte observation des réformes exigées par l'Union européenne. Ses concepteurs admettent qu'une telle politique peut être source de souffrances, mais ils estiment qu'elle immunisera la société contre des douleurs plus vives encore. Une privatisation complète de l'agriculture est selon eux nécessaire. La petite agriculture de subsistance doit par ailleurs être éliminée de façon à favoriser la constitution de grandes exploitations agricoles, capables de pratiquer une agriculture intensive et de se conformer aux normes de productivité européennes. Le programme ainsi formulé a pour porte-drapeaux l'Union des forces démocratiques (Sajuz na Democraticnite Sili, SDS) en Bulgarie et le Parti national paysan - Chrétien démocrate (Partidul National Taranesc - Crestin Democrat, PNTCD) en Roumanie. Les formations qui représentent une minorité nationale tendent aussi à s'en faire les défenseurs : lorsqu'il s'agit de nouer un dialogue avec des partis à vocation majoritaire, l'Union démocratique des Magyars de Roumanie (Uniunea Democrata Maghiara din România, UDMR) affiche des positions intégrationnistes ; attaché à défendre les intérêts de la minorité turcophone de Bulgarie, le Mouvement des droits et libertes (Dvisenie za Pravata i Svobodie, DPS) conçoit la même inclination, mais il se montre plus pragmatique et accepte toute forme de partenariat politique qui peut servir son propos ${ }^{16}$.

Les défenseurs du programme conciliatoire proposent un moyen terme entre les options jusqu'alors envisagées. Ils se montrent favorables aux réformes dans la mesure où elles conditionnent l'obtention de crédits communautaires, mais ils demandent qu'elles soient limitées au strict nécessaire. Ils recherchent en somme un dosage optimal entre soumission aux contraintes externes et préservation des équilibres internes. Ils préconisent des formes d'organisation intermédiaires, qui échappent aux principes collectivistes et à la planification, mais qui demeurent - au moins partiellement - entre les mains de l'État. Ils défendent ces vues à la tête du BSP et dans l'entourage de Ion Iliescu.

Les programmes ainsi définis ne suffisent pas à expliquer les résultats électoraux contrastés que les formations politiques obtiennent dans les campagnes. Ils n'influent sur le vote des paysans que dans la mesure où ils participent d'un même "fondu-enchaîné" caractérisée plus haut. Cette logique introduit un facteur de variation à deux niveaux emboîtés.

Au niveau macro-institutionnel, il convient d'examiner les modalités selon lesquelles les programmes sont mis en œuvre. Les réformes introduites dans l'agriculture sont largement tributaires des relations agraires instaurées sous le régime communiste : selon que les paysans ont entretenu un rapport harmonieux ou tendu avec le pouvoir, la décollectivisation peut obéir à des logiques diversifiées.

${ }^{16}$ Cf. Roger (Antoine), "Perspectives d'intégration à l'Union européenne et formation des systèmes de partis en Europe orientale ", Politique europeenne, (3), 2001. 
Les variations ainsi relevées s'emboîtent dans le niveau micro-institutionnel. La logique de la décollectivisation conditionne la diffusion des mots d'ordre politiques auprès des paysans. Les programmes agraires sont récupérés par des intermédiaires locaux puis adaptés aux nouvelles structures locales selon différentes modalités.

\section{LA LOGIDUE DE LA DÉCOLLECTIVISATION}

En Bulgarie comme en Roumanie, une politique conciliatoire est menée immédiatement après la chute du régime communiste. Encore ne produit-elle pas des effets identiques dans les deux cas : marquées par leurs expériences passées, les paysanneries ne réagissent pas sur le même mode au changement de contexte économique.

En Bulgarie, les relations agraires ont conservé un caractère harmonieux jusqu'à la fin du régime communiste. Les paysans ne manifestent donc aucun empressement à quitter les structures collectives. La politique conciliatoire peut être appliquée selon une logique de transvasement : les modes de culture et les normes de travail hérités de l'économie socialiste sont conservés et orientés simplement vers des récipients institutionnels plus conformes aux exigences de l'Union européenne.

En Roumanie, les autorités communistes ont durci leur politique agraire dans les années 1980. Après l'effondrement du régime, les paysans entendent s'affranchir de toute contrainte institutionnelle : ils s'empressent de démanteler les structures collectives et s'orientent vers la petite agriculture privée. La politique conciliatoire obéit dès ce moment à une logique de drainage : elle consiste à former de nouveaux récipients institutionnels et à y attirer des propriétaires pour lors dispersés.

\section{Une logique de transvasement}

Dans la Bulgarie communiste, la collectivisation s'opère sur un double mode. À la fin des années 1940, des fermes d'État sont constituées à partir des grands domaines expropriés; elles couvrent $8 \%$ de la surface agricole utile. 3290 coopératives (TKZS) sont parallèlement formées dans lesquelles les paysans restent nominalement propriétaires de leurs terres mais sont sommés de les travailler collectivement : $90 \%$ de la surface agricole utile est exploitée selon ce principe.

Dans les coopératives, un lopin auxiliaire d'un demi-hectare est mis à la libre disposition de chaque paysan. Il sert le plus souvent à pratiquer une agriculture de subsistance. Un rapport de complémentarité est toutefois observé entre les cultures collectives et les cultures individuelles pratiquées sur les 
lopins auxiliaires. La production des lopins couvre les besoins alimentaires des paysans et permet d'orienter toutes les récoltes tirées des terres collectives vers les circuits de distribution nationaux. En retour, la coopérative fournit les engrais chimiques, les semences et le matériel agricole nécessaires à l'exploitation des lopins. Elle aménage des pâtures complémentaires pour le bétail que les paysans élèvent sur leur lopin et offre des services vétérinaires ${ }^{17}$. Lorsque la production des lopins individuels outrepasse les besoins des paysans, les surplus sont vendus à l'État sur une base contractuelle et réinsérés dans le secteur socialiste. En guise de paiement, les paysans reçoivent de la nourriture pour leur bétail ${ }^{18}$.

L'imbrication étroite des cultures individuelles et des cultures collectives permet aux autorités d'entretenir de bonnes relations avec les masses rurales. Si la paysannerie tire sa subsistance des lopins auxiliaires, cette orientation est intégrée au fonctionnement des coopératives plutôt qu'elle n'est conçue comme une échappatoire. Quelques réformes sont bien introduites dans les campagnes mais elles visent à améliorer la productivité des exploitations plutôt qu'à mettre les paysans au pas. Dans les années 1960, une politique de regroupement des unités de production est engagée : les coopératives sont fusionnées et ramenées au nombre de 930. Au début des années 1970, le Comité central décide de soumettre l'agriculture aux méthodes de production industrielles; un effort de mécanisation est engagé et chaque unité est poussée à se spécialiser dans un type de culture : les coopératives cèdent ainsi la place à 160 complexes agro-industriels couvrant 2400 hectares en moyenne. La réforme se solde par un échec économique. À la fin des années 1970, les autorités décident donc de revenir sur leurs pas : les complexes agro-industriels sont démantelés et des coopératives de taille plus réduite sont formées. En 1986 , les unités de production sont finalement ramenées à leurs dimensions initiales.

Les réformes successivement mises en œuvre portent sur l'étendue et l'affectation des terres collectives mais ne remettent jamais en cause l'équilibre général observé entre ces terres et les lopins auxiliaires; elles ne portent donc pas atteinte à l'assise du Parti communiste dans les campagnes. Après l'effondrement du régime, les paysans ne manifestent aucune ardeur à démanteler les coopératives. Lorsque le Parlement en vient à voter, au printemps 1991, une Loi sur la propriété et l'utilisation des terres affermées, il n'a garde de détruire l'édifice institué. 4800 territoires sont simplement délimi-

${ }^{17}$ Cf. Kaneff (Deema), " Responses to "Democratic" Land Reforms in Bulgaria ", in Abrahams (Ray), ed., After Socialism : Land Reform and Social Change in Eastern Europe, Oxford : Berghahn Books, 1996, pp. 8687.

18 Cf. Creed (Gerald W.), "Deconstructing Socialism in Bulgaria n, in Burawoy (Michael), Verdery (Katherine), eds., op. cit., p. 233. 
tés. Des Commissions agraires sont chargées d'y procéder à la restitution des terres. Leurs activités sont rigoureusement encadrées : la loi précise qu'un individu ne peut se voir attribuer plus de 30 hectares (la limite est rabaissée à 20 hectares dans les zones de culture intensive). Les terres restituées doivent être consacrées à l'agriculture sous peine de sanctions fiscales. Elles ne peuvent être vendues avant un délai de trois ans. Seuls des membres de la famille, des propriétaires voisins ou des paysans qui la louaient peuvent ensuite s'en porter acquéreur. L'État et la municipalité ont la possibilité de déroger à cette règle et d'exercer un droit de préemption ${ }^{19}$. Une Loi sur les coopératives vient compléter le dispositif. Elle autorise les paysans à enregistrer de nouvelles coopératives bénéficiant d'un statut réaménagé. Les propriétaires doivent désormais recevoir des dividendes à proportion de la terre qu'ils engagent dans la coopérative et peuvent reprendre leur liberté quand bon leur semble.

À peine ces lois sont-elles entrées en vigueur que des élections sont organisées. Le BSP n'obtient que $36,20 \%$ des voix, résultat insuffisant pour prétendre conduire la législature. Le SDS ne réalise pas un meilleur score : il ne recueille que $34,34 \%$ des suffrages exprimés. Avec l'appui du DPS (7,55 \% des voix), il parvient cependant à former un gouvernement d'orientation intégrationniste.

Les nouveaux dirigeants ne goûtent guère le dispositif conciliatoire mis en place par leurs prédécesseurs. Ils y voient un moyen ourdi par les héritiers du régime communiste pour défendre leurs positions acquises dans les campagnes. En mars 1992, ils amendent par conséquent la Loi sur la propriété. Les restrictions quantitatives sont levées, ainsi que les obstacles posés à la revente des terres. La Loi sur les coopératives est pareillement remaniée : aux termes du nouveau texte, les terres doivent obligatoirement être redistribuées en considération des découpages établis avant la collectivisation ; il n'est plus question de transformer directement les titres de propriété en titres de participation à une nouvelle coopérative.

Afin d'accélérer le mouvement de décollectivisation, le gouvernement met en place un double dispositif. Des Commissions agraires sont tout d'abord chargées d'enregistrer les réclamations et d'examiner les documents justificatifs des familles désireuses de recouvrer la pleine propriété de leurs terres. Chaque commission est composée d'un président, de trois arpenteurs et de deux agronomes, tous désignés par le Conseil général. Avant d'entrer en exercice, une commission doit recevoir du ministère de l'Agriculture un agrément

$19 \mathrm{Cf}$. Creed (Gerald W.), "An Old Song in a New Voice : Decollectivization in Bulgaria ", in Kideckel (David A.), ed., op. cit., p. 27 ; Kopeva (Diana), Mishev (Plamen), Howe (Keith), " Land Reform and Liquidation of Collective Farm Assets in Bulgarian Agriculture : Progress and Prospects n, Communist Economies and Economic Transformation, (6), 1994, p. 204. 
qui peut ensuite lui être retiré à tout moment. Le gouvernement entend ainsi s'appuyer sur des hommes de confiance et contourner l'autorité que peuvent exercer localement les anciens responsables de coopératives.

Des Comités de liquidation comprenant quatre à six membres sont ensuite affectés à la tête de chaque coopérative en remplacement de l'ancienne direction. Ils ont pour mission de vendre le matériel agricole et les bâtiments puis de redistribuer les sommes collectées sous forme d'actions (dyalov kapital). Les membres des comités sont désignés par le Conseil régional. Le plus souvent extérieurs au village, ils sont choisis parmi les militants et les sympathisants affichés du SDS. Leur salaire est prélevé sur les revenus de la coopérative. Dans nombre localités, les paysans dénoncent le caractère occulte de leurs transactions et font état de malversations. En 1993, la loi est révisée en conséquence: les villageois obtiennent un droit de contrôle sur la composition des Comités de liquidation. Leur pouvoir demeure toutefois limité : le ministère de l'Agriculture conserve la possibilité de confirmer dans leurs fonctions les membres qui ont été récusés au niveau local ${ }^{20}$.

Si le nouveau dispositif apporte satisfaction aux membres du SDS, il suscite quelques dissensions au sein du DPS : les Comités de liquidation s'acquittent très rapidement de leur mission dans les régions agricoles où la population turcophone est majoritaire. Spécialisées dans la production du tabac, les coopératives visées n'ont guère été mécanisées ; la revente de leur matériel ne pose donc aucune difficulté. Les familles turcophones n'ayant généralement aucun titre de propriété à faire valoir, les Commissions agraires n'ont guère de dossier à traiter. Les exploitations de tabac non rentables sont simplement démantelées et leur main d'œuvre est licenciée sans autre forme de procès. Les villages turcophones comptent dès ce moment 25 à $90 \%$ de chômeurs. Au nom de leurs mandataires, plusieurs représentants du DPS protestent contre la politique agricole du gouvernement. En octobre 1992, la direction du parti finit par se rallier à leurs vues et par refuser de collaborer plus avant avec le SDS ${ }^{21}$. Ce revirement provoque la chute du gouvernement en place. Un cabinet est aussitôt constitué sous l'impulsion du DPS, avec l'appui du BSP.

Une politique d'accommodement est alors adoptée. Les autorités encouragent la formation de nouvelles coopératives, mais elles ne reviennent pas sur

20 Cf. Kaneff (Deema), "Developing Rural Bulgaria ", Cambridge Anthropology, 18 (2), 1995 ; Kaneff (Deema), "Responses to "Democratic" Land Reforms in Bulgaria " (art. cit.), pp. 89-90; Billaud (M.), "Les campagnes bulgares : le grand chambardement pour quels résultats ? ", in Rey (Violette), éd., Les nouvelles campagnes de l'Europe centre orientale, Paris : CNRS Editions, 1996 ; Kopeva (Diana), Mishev (Plamen), Howe (Keith), art. cit., Pp. 208, 213.

${ }^{21}$ Meurs (Mieke), Begg (Robert), art. cit. ; Bates (Daniel), "Uneasy accommodation : ethnicity and politics in Rural Bulgaria ", in Kideckel (David A.), ed., op. cit., p. 146. 
les amendements préalablement votés pour faciliter la revente des terres. Le gouvernement se plait à expliquer qu'il laisse les paysans libres de leur choix. Dans les faits, les terres sont très largement réinsérées dans les nouvelles cooperatives : en 1993, seulement 9,7\% des paysans exploitent individuellement leur terre $\mathrm{e}^{22}$.

Les relations agraires instaurées en Bulgarie pendant la période communiste influent fortement sur le cours des réformes adoptées dans les années qui suivent le changement de régime : elles permettent un transvasement des structures collectives dans des cadres institutionnels réformés en réponse aux exigences de l'Union européenne. Une comparaison avec la trajectoire empruntée en Roumanie permet de bien saisir la portée de telles caractéristiques. Le Parti Communiste Roumain n'étant jamais parvenu à instaurer un rapport harmonieux entre les cultures collectives et les cultures individuelles, il a dû recourir à des méthodes de plus en plus coercitives pour soumettre les campagnes à ses volontés. Le régime à peine effondré, les paysans s'affranchissent des structures collectives et se replient sur des petites parcelles privées impropres à la pratique d'une agriculture intensive. La politique conciliatoire ne peut alors se traduire par un simple transvasement institutionnel; elle consiste plutôt à drainer les paysans vers des formes de coopération plus poussées.

\section{Une logique de drainage}

En octobre 1945, une première réforme agraire est introduite en Roumanie. Elle touche les grands propriètaires fonciers qui possédaient plus de 50 hectares; avec les terres ainsi libérées, des "entreprises agricoles d'État" (Intreprideri Agricole de Stat) sont constituées. Au même titre que les entreprises industrielles, elles sont soumises au plan : des objectifs de production et des objectifs de rendement leur sont imposés ; un parc de machines propre et des conducteurs d'engins agricoles (tractoristi) leur sont affectés; une part des bénéfices réalisés sert à constituer un fonds de salaire et à rémunérer les employés.

En 1949, les paysans qui possèdent de 5 à 10 hectares sont à leur tour expropriés. 940 ooo hectares sont ainsi accaparés qui viennent élargir le terrain des entreprises agricoles d'État. Les petits paysans qui possèdent moins de 5 hectares sont pour leur part poussés à intégrer des "coopératives agricoles de production" (Cooperativa Agricola de Productie). Si ces nouvelles unités de production sont soumises à un régime de planification, elles ne sont pas contrôlées

\footnotetext{
22 Commission Euxopéenre, "Agricultural Situation and Prospects in the Central and Eastem Europe Countries : Bulgaria " (art. cit.) ; Meurs (Mieke), spreeuw (Darren), " The evolution of agrarian institutions in Bulgaria : markets, cooperatives and private farming 1991-1994 ", in Jones (Derek C.), Miller (Jeffrey), eds., The Bulgarian Economy : Lessons from Reform during Early Transition, Aldershot : Ashgate, 1997 ; Yarmal (Brent), " De-collectivization of Bulgarian Agriculture ", Land Use Policy, (11), 1994.
} 
aussi étroitement que les entreprises agricoles d'État. Chacune se voit imposer des objectifs annuels mais reste libre de les atteindre par les moyens qui lui conviennent. Quelques normes d'organisation sont simplement édictées. Les "paysans coopérateurs" (tarani cooperatorii) sont rassemblés par groupes de 15 dans des brigades ; ils travaillent ensemble les terres collectives. Les coopératives ne disposent pas d'un parc de machines propre; elles doivent louer leurs tracteurs à des "stations de mécanisation agricole" (Statie de Mecanisare Agricola). Les paysans coopérateurs ne travaillent sur les terres collectives que de 7 heures du matin à 3 heures de l'après-midi ; ils peuvent occuper le reste de leur journée à des activités complémentaires. Ils disposent à cet effet de lopins individuels. Un lopin n'est pas assimilable à une propriété privée : il est simplement alloué par la coopérative et ne peut être vendu ni transmis par héritage. Sa superficie est de 1500 mètres carrés en moyenne. Ce chiffre dissimule toutefois quelques disparités : les dirigeants de coopératives disposent de lopins plus vastes, couvrant jusqu'à 14 hectares. Dans tous les cas, les récoltes tirées du lopin individuel peuvent être vendues sur le marché privé.

Dans l'optique des dirigeants communistes, les lopins individuels doivent amener les masses rurales à travailler les terres collectives sans les brusquer ni les couper totalement de leurs pratiques accoutumées : selon ce calcul, le paysan coopérateur doit se montrer docile dès l'instant qu'il reste libre de se livrer à de petites cultures de subsistance sur son lopin individuel. Les intéressés déjouent rapidement ces prévisions en consacrant toute leur énergie à leur lopin et en se derobant au travail collectif. Plusieurs réformes sont successivement introduites pour les mettre au pas. Elles ne sont jamais payées de succès. Dans les années 1980, les autorités se résolvent à adopter des mesures radicales. Elles décident de confisquer les lopins individuels et de reloger les paysans dans des blocs standardisés. À titre de substitution, elles prévoient d'aménager des jardins de 80 à 90 mètres carrés dans le périmètre de construction et de les attribuer collectivement aux habitants d'un même bloc. Les changements intervenus en 1989 interrompent la mise en œuvre de cette politique ${ }^{23}$.

Libérés de la menace qui pesait sur eux, les paysans se réapproprient spontanément les terres des coopératives. Lopins individuels et terres collectives confondues, 3 millions d'hectares passent en régime de propriété privée au début de l'année 1990 . Cette mutation brutale perturbe les circuits de distribu-

23 Roger (Antoine), "Relations agraires et relations de pouvoir dans la Roumanie communiste : les Coopératives Agricoles de Production comme enjeu politique ", Revue d'Histoire moderne et contemporaine, 49 (2), avril-juin 2002. 
tion. Les paysans issus des coopératives dissoutes produisent pour leur propre consommation. Ils stockent les surplus ou les écoulent sur des petits marchés de proximité qui échappent totalement au contrôle de l'État. En conséquence, plusieurs grandes villes connaissent des difficultés d'approvisionnement en huile, sucre et farine durant l'été $1990^{24}$.

Les autorités sont désarmées. Elles sont placées devant le fait accompli. Sauf à provoquer un soulèvement des campagnes, elles ne sauraient réclamer aux paysans de reconstituer les coopératives. Elles doivent se contenter de régulariser la situation. En février 1991, une Loi sur l'Agriculture et les ressources agraires (Loi 18/1991) est ainsi votée.

Les coopératives sont officiellement dissoutes. La terre est redistribuée aux anciens propriétaires. Il n'est pas question cependant de redonner vie à la structure agraire de l'entre-deux-guerres. Parmi les paysans qui se sont emparés d'une parcelle, beaucoup sont issus de familles qui n'avaient pas ou peu de terres au moment de la collectivisation. Pour éviter toute frustration et tout affrontement, il est nécessaire de leur accorder des titres de propriété. Un correctif doit alors être apporté au principe de la simple restitution. Les titres de propriété anciens sont rabotés en application d'un système de plafonnement : la superficie récupérée au bénéfice de la réforme est limitée à 10 hectares par famille. Tout paysan qui a travaillé plus de 3 ans dans une coopérative agricole de production reçoit une parcelle de 0,5 à 1 hectare. Les mécaniciens et tous les cadres intermédiaires employés sur place bénéficient du même régime. Lorsque la quantité de terres fournie par la coopérative dissoute ne permet pas de satisfaire toutes les attentes, un coefficient de réduction permet d'opérer une ponction sur les parcelles distribuées aux anciens propriétaires. À l'inverse, lorsqu'un excédent de terre est disponible, il est distribué par lots à des familles qui n'étaient pas engagées dans la coopérative mais qui souhaitent s'adonner à une activité agricole. La cession des terres reçues à titre de restitution est autorisée, mais elle est soumise à l'approbation d'une commission locale : composée de fonctionnaires, cette instance doit veiller qu'aucune propriété individuelle ne dépasse les 100 hectares. Pour utiliser le matériel des coopérati-

24 Cf. Hirschausen (Beatrice Von), "Réforme agraire en Roumanie : nouveaux rapports au territoire ", Cahiers Nantais, (43), 1995, pp. 181-184 ; Hirschausen (Bëatrice Von), Les nouvelles campagnes roumaines : paradoxes d'un " retour " paysan, Paris : Belin, 1997, p. 45 ; Sivignon (Michel), "La décollectivisation des campagnes roumaines ", Economie Rurale, (214-215), 1993 ; Lhomel (Edith), "La décollectivisation des campagnes roumaines : incertitudes et enjewx ", Revue des Etudes Comparatives Est-Ouest, (3). 1995, p. 132 ; Kideckel (David A.), " Once Again, the Land : Decollectivization and Social Conflict in Rural Romania n, in Anderson (David), De Soto (Hermine), eds., The Curtain Rises : Rethinking Culture, Ideology and the State in Eastern Europe, Atlantic Highlands : Humanities Press, 1992, pp. 92-98. 
ves démantelées, les paysans sont invités à former des associations agricoles. Lorsque aucune association n'est créée, le matériel est vendu aux enchères ${ }^{25}$.

$\mathrm{Si}$, en Roumanie comme dans toutes les anciennes démocraties populaires, un équilibre est recherché entre justice réparatrice et justice distributive, le fléau de la balance penche résolument vers la seconde ${ }^{26}$. Pressées par la base, les autorités roumaines reconstituent les titres de propriété plutôt qu'elles ne les restituent. Elles ne reviennent pas au statu quo ante mais introduisent indirectement une réforme agraire. 3800 coopératives sont liquidées au total ; 9 millions d'hectares sont redistribués à 5,6 millions de propriétaires. $66 \%$ de la surface agricole totale sont désormais couverts par des propriétés inférieures à 2 hectares. La taille moyenne des propriétés est de 1,8 hectares ${ }^{27}$.

Les autorités ne renoncent pas à pousser les paysans issus des coopératives agricoles d'État vers des formes d'agriculture plus intensives. Les stations de mécanisation agricole héritées de la période communiste sont ainsi transformées en sociétés commerciales et rebaptisées Agromec. Au nombre de 520, elles détiennent $50 \%$ du parc national de tracteurs. Elles sont placées sous le contrôle direct du ministère de l'Agriculture. Les capitaux qui y sont investis

${ }_{25}$ Topor (Gabriel), " Romania : The Postcommunist Land Law ", Report on Eastern Europe, 30/08/91 ; Constantinescu (Nicolae N.), " The New Agrarian Land Law in Romania and Its Impact on Sustainable Agriculture ", Revue Roumaine des Sciences Economiques, 36 (2), 1992 ; Rey (Violette), Ianos (Ioan), Leclerc (Brigitte), "Les campagnes roumaines en début de transition, 1990-1991", Bulletin de l'Association des Géographes Français, (1), 1992 ; Otiman (P. I.), Agricultura României la cumpana dintre mileniile II si III, Helicon, Timisoara, 1994, pp. 51-70 ; Verdery (Katherine), " Decollectivization, Democracy and the "LawGoverned State" : A View from the Village ", Sfera Politicii, (23), 1994 ; Verdery (Katherine), What Was Socialism and What Comes Next, Princeton : Princeton University Press, 1996, p. 37 ; Stewart (Michael), "We should build a statue to Ceausescu here : the trauma of decollectivisation in two Romanian villages ", in Bridger (Sue), Pine (Frances), eds., Surviving Post Socialism : Local Strategies and Regional Responses in Eastern Europe and the Former Soviet Union, London : Routledge, 1998 ; Cartean (C.), Cretoiu (V.), " Perceptia tranzitiei în mediul rural ", Sociologie Româneasca, (3), 1993.

Les entreprises agricoles d'État employaient dans la période communiste une main-d'œuvre salariée. Elles n'attribuaient pas de lopins individuels; elles n'étaient donc pas soumises aux mêmes mesures coercitives que les coopératives agricoles de production. Pour cette raison même, elles demeurent bien assises sur leurs fondations après la chute de Ceausescu : elles n'ont pas été démembrées par la base ; leurs terres n'ont pas fait l'objet d'une redistribution spontanée. Les salariés ont simplement ralenti leur rythme de travail après qu'un terme a été mis aux mesures de planification. Ils n'ont pas cherché à imposer un nouveau mode d'organisation interne. Le législateur conçoit de privatiser partiellement les entreprises agricoles d'État : il leur attribue un statut de société commerciale assez comparable à celui des SARL françaises. $25 \%$ des parts sont octroyées aux anciens propriétaires ou à leurs héritiers ; $30 \%$ servent à constituer un fond de propriété privée et reviennent de fait aux salariés ; les $45 \%$ restants sont mis en vente. Toute personne physique ou morale peut légalement les acquérir. En l'absence d'investisseurs intéressés, c'est l'État qui les accapare en fait. Les sociétés commerciales sont ainsi placées sous le contrôle direct du Ministère de l'Agriculture. Elles restent soumises à des directives nationales (cf. Henry (David C.), " Reviving Romania's Rural Economy ", Radio Free Europe / Radio Liberty - Research Report, 3 (7), 1994, p. 21).

${ }^{26}$ Maurel (Marie-Claude), La transition post-collectiviste : Mutations agraires en Europe centrale , Paris : L'Harmattan, 1994, pp. 348-349.

${ }^{27}$ Hirschausen (Béatrice Von), op. cit., p. 67. 
sont publics à $100 \%$; il est prévu qu'ils se diversifient progressivement. Sur la même base, une société Semrom est créée dans chaque département (judet) : elle fournit les paysans en engrais et en semailles. Le réseau de collecte des céréales est maintenu sur pied ; il est transformé en trust commercial à participation publique majoritaire et prend le nom de Romcereal. Disposant de nombreuses bases locales, il achète les céréales en considération des besoins nationaux et assure leur bonne diffusion sur l'ensemble du territoire.

En articulation avec ces trois institutions, une Loi sur les sociétés agricoles et autres formes d'association agricoles (loi 36/1991) est votée en avril 1991. Elle vise à édifier des structures collectives intermédiaires qui, sur la base du volontariat, regroupent les petites exploitations individuelles en unités agricoles productives et rentables. Elle établit une distinction entre associations familiales et sociétés agricoles. Les premières reposent sur un accord informel entre paysans ; elles ne disposent d'aucune personnalité juridique. Les secondes doivent déposer leurs statuts en préfecture. Les paysans qui fondent une société agricole réalisent leurs investissements et travaillent la terre en commun, mais chacun commercialise sa récolte comme il l'entend. Toute société agricole est gérée par un conseil d'administration ; son capital est divisé en parts ; ses membres sont libres de la quitter à tout moment.

Les exploitants individuels ne peuvent entrer en relation avec les stations Agromec et Semrom ou avec les bases Romcereal que par l'intermédiaire d'une société agricole. Ils obtiennent de cette façon du matériel agricole, des semences, des engrais et des produits phytosanitaires à des prix préférentiels.

Agromec, Semrom et Romcereal fonctionnent de façon emboitée au niveau local : pour chaque campagne agricole, la société Romcereal reçoit un crédit du ministère de l'Agriculture ; elle paie par avance aux stations Agromec et Semrom les services rendus aux sociétés agricoles ; en échange de cette assistance, les paysans concernés lui livrent une partie de leur récolte ${ }^{28}$.

À l'inverse des coopératives bulgares, les sociétés agricoles ne peuvent regrouper dès l'abord l'ensemble de la paysannerie. Avant qu'elles ne soient mises sur pied, les populations rurales se sont polarisées en deux ensembles, l'un et l'autre attachés à des cultures individuelles.

Un premier ensemble est formé par les grands paysans qui ont pu amorcer une dynamique d'accumulation et s'essayer rapidement à l'agriculture intensive. Sur l'ensemble des propriétaires, seulement $5 \%$ ont les moyens de réaliser des investissements à moyen terme. Ils parviennent à orienter la plus grande partie de leur récolte vers les marchés urbains et réalisent des bénéfices substantiels qui leur permettent d'élargir encore leur propriété. Les anciens directeurs de coopératives agricoles de production forment le plus gros de leur 
effectif. Sous le régime communiste, ils ont pu accumuler quelque argent dans l'exercice de leurs fonctions ${ }^{2}$.

Un second ensemble est constitué par la petite paysannerie. Les deux tiers des paysans recensés au début des années 1990 ne forment aucun projet d'investissement ; ils consacrent la quasi-totalité de leur récolte à leur propre consommation et se procurent les biens qu'ils ne peuvent produire eux-mêmes par un système de troc. Ils se tiennent à l'écart des réseaux de distribution institutionnels ${ }^{30}$.

Coexistant dès l'origine avec des régimes de propriété différenciés, les sociétés agricoles ne peuvent avoir le même statut que les coopératives bulgares. Elles ne visent pas à dissuader les paysans de s'orienter vers des cultures individuelles mais à convaincre les propriétaires qui pratiquent ces cultures d'y renoncer et de revenir à des méthodes de travail en commun. Les grands paysans ne sont pas visés : ils se livrent déjà à des cultures intensives et il n'est pas question de les amener à de nouvelles pratiques. Les petits paysans qui s'adonnent à des cultures de subsistance sont une cible prioritaire. Leurs parcelles ne sont guère étendues isolément, mais elles couvrent dans leur ensemble une surface importante et doivent à ce titre être réinsérées dans les circuits de distribution nationaux.

Entre les trajectoires bulgare et roumaine des écarts significatifs peuvent être relevés. Dans la période communiste les structures agraires semblent certes présenter des contours identiques : une division est observée entre des fermes d'État et des coopératives dans lesquelles les paysans disposent librement d'une petite parcelle. Mais ces lignes extérieures dissimulent des différences majeures : si les lopins individuels et le secteur socialiste sont étroitement imbriqués en Bulgarie, ils se développent sur un mode antagonique en Roumanie ; les relations agraires peuvent rester paisibles dans le premier cas tandis qu'elles ne cessent de se durcir dans le second. Cette bifurcation ex-

29 Cf. Mihailescu (Vintila), Nicolau (Viorica), " La "transition " entre holisme et individualisme ", Romanian Journal of Sociology, 6 (1), 1995, p. 73 ; Marginean (Ion), " Rural Areas and Social Reform in Romania ", Romanian Journal of Sociology, 6 (1), 1995 ; Sampson (Steven L.), " Money without Culture, Culture without Money : Eastern Europe Nouveaux Riches ", Anthropological Journal of European Culture, (2), 1993 ; Verdery (Katherine), What Was Socialism and What Comes Next (op. cit.), p. 137 ; Verdery (Katherine), " Disambiguating ownership : Rights and power in Transylvania's decollectivation ", in Hann (C. M.), ed., Property Relations : Reviewing the Anthropological Tradition, Cambridge : Cambridge University Press, 1998, pp. 102-103.

$3^{\circ}$ Cf. Hirschausen (Béatrice Von), op. cit., pp. 66, 73, 185, 200-201; Hirschausen-Leclerc (Béatrice Von), art. cit., p. 322 ; Kideckel (David A.), "Once Again, the Land " (art. cit.), p. 88 ; Kideckel (David A.), " Populatia rurala si autoritatea în noua Românie ", Sfera Politici, (6), 1993 ; Fulea (Maria), " Structura socio-economica a populatiei rurale în perioada de tranzitie la economia de piata ", in Florian (Violeta), Fulea (Maria), Sarbu (Aurelia), eds., Satul românesc contemporan, Bucarest : Editura Academiei, 1996 ; Commission Européenne, "Agricultural Situation and Prospects in the Central and Eastem Europe Countries : Romania " (art. cit.). 
plique les différences observées ensuite au moment de la décollectivisation. Dans les deux pays, une politique conciliatoire est bien mise en œuvre qui permet de mettre sur pied des structures agraires intermédiaires - éloignées des structures collectivistes aussi bien que des règles de la libre concurrence. Mais les résultats obtenus sont loin d'être identiques : en Bulgarie, il est possible de former de nouvelles coopératives par un simple réaménagement institutionnel dans la mesure où les paysans demeurent inscrits en grand nombre dans les structures héritées de la période communiste ; en Roumanie, les sociétés agricoles ne peuvent connaître un succès aussi prompt car les paysans ont fui les structures collectives pour se consacrer individuellement à leur parcelle.

Les divergences ainsi relevées forment un contraste avec le caractère stéréotypé des projets que les partis politiques élaborent en réponse aux pressions exercées par l'Union Européenne. Si les programmes anti-intégrationniste, intégrationniste et conciliatoire défendus au niveau national présentent les mêmes contours en Bulgarie et en Roumanie, ils ne sont pas diffusés sur le même mode dans les campagnes : dès lors que les paysans sont insérés dans des relations agraires différenciées au niveau macro-institutionnel, il ne peuvent être mobilisés par des moyens identiques au niveau micro-institutionnel. Les intermédiaires locaux des partis politiques doivent adapter les programmes aux réalités locales.

\section{LE TRAVAIL DES INTERMÉDIAIRES LOCAUX}

Dans les villages bulgares et roumains, les militants intégrationnistes, antiintégrationnistes et conciliatoires invitent les paysans à soutenir le programme agraire défini par leur parti. Ils travaillent ainsi à collecter des suffrages dans la perspective des prochaines élections nationales en même temps qu'ils servent leurs propres ambitions politiques au niveau local. Les méthodes qu'ils utilisent diffèrent selon que la décollectivisation a obéi à une logique de transvasement ou à une logique de drainage. Dans le premier cas, les paysans sont engagés en grand nombre dans des coopératives réformées ; durant les années qui suivent le changement de régime, les intermédiaires locaux doivent se glisser dans ces structures et les faire fonctionner dans un sens qui serve leur propos ; il s'agit pour eux d'acclimater les paysans à des pratiques conformes au programme défendu. Dans le second cas, les intermédiaires locaux ont affaire à une paysannerie dispersée ; en utilisant les structures coopératives de manière circonstanciée, ils s'efforcent de prouver que les comportements préconisés par leur programme agraire sont les plus porteurs ; ils entendent ainsi montrer l'exemple et inciter les paysans à suivre la voie qu'ils indiquent : les sociétés agricoles sont pour eux des instruments de démonstration. 
Les intermédiaires locaux se heurtent à des obstacles différents selon qu'ils se trouvent placés dans l'une ou l'autre configuration. Leurs difficultés sont, dans tous les cas, amplifiées lorsqu'une concurrence s'instaure entre plusieurs types de coopératives à l'intérieur d'un même village : chaque intermédiaire s'efforce alors de démontrer la supériorité de son programme et d'attirer les paysans qui adhèrent pour lors à un autre modèle ; dès qu'une coopérative connaît quelques déboires, ses rivales s'efforcent d'en tirer parti. Les intermédiaires conciliatoires se trouvent dès l'abord en position de force dans la mesure où ils s'appuient sur des structures agraires conçues pour servir leur propos. Une fois installés, ils sont néanmoins amenés à prendre quelque licence avec le programme agraire que défend leur parti. Leur succès immédiat n'est pas le gage d'une implantation politique pérenne.

\section{Un travail d'acclimatation}

En Bulgarie, la structure agraire présente durant plusieurs années un caractère homogène. La majorité des propriétaires se refuse à travailler la terre isolément et préfère se maintenir dans les nouvelles coopératives ${ }^{31}$. Les intermédiaires locaux utilisent ces coopératives pour transformer les habitudes des paysans, dans l'espoir que leur vote sera orienté en conséquence. Les obstacles rencontrés sont plus ou moins difficiles à surmonter selon que l'objectif est de diffuser un programme anti-intégrationniste, intégrationniste ou conciliatoire.

Les intermédiaires anti-intégrationnistes sont les anciens responsables locaux du Parti Communiste Bulgare. Ils intègrent les conseils d'administration de coopératives constituées pour y défendre leurs vues : ils y louent les mérites des structures agraires instaurées sous le régime communiste et expliquent que les représentants corrompus du SDS ont travaillé à leur démembrement contre la volonté des paysans, avec le seul soutien de puissances occidentales attachées à conquérir de nouveaux marchés. Les malversations observées suffisent selon eux à prouver le caractère néfaste des réformes engagées. Pour assainir les relations agraires, il convient d'écarter de toutes les coopératives les responsables avides de gains individuels et de les remplacer par les dirigeants qui ont fait autrefois la preuve de leur désintéressement ${ }^{32}$.

Ce discours trouve quelque écho dans les campagnes bulgares mais aucun paysan n'y adhère totalement. Les populations rurales lui prêtent l'oreille dans la mesure où le changement de régime leur parait piloté par des élites urbaines distantes et peu soucieuses des problèmes qui affectent le secteur agricole.

${ }^{31}$ Le glissement vers l'agriculture individuelle ne s'opère que dans la seconde moitié des années 1990 (Nemenyi (Agnes), " Restructurare rurala si autosubzistenta gospodariilor familiale : studiu comparativ între România si Bulgaria ", Sociologie româneasca, (3), 1999, p. 72).

${ }^{32}$ Creed (Gerald w.), " An Old Song in a New Voice " (art. cit), p. 229. 
L'influence politique qu'elles pouvaient exercer sous le régime communiste leur parait forte en comparaison : par des pressions informelles, les paysans parvenaient à faire valoir leurs intérêts ; le nouveau régime leur accorde le droit d'élire librement leurs représentants, mais il les prive de relais jugés plus efficaces. Les intermédiaires anti-intégrationnistes s'appuient sur ce sentiment diffus, mais ils n'en retirent que des avantages limités : les paysans sont satisfaits de pouvoir vendre leurs récoltes avec quelques perspectives de profit plutôt que de devoir les livrer systématiquement à l'État. S'ils récriminent contre les inconvénients relatifs du nouveau régime, ils ne manifestent pas la volonté de restaurer un système économique centralisé33.

Faute d'obtenir des soutiens fermes, les intermédiaires anti-intégrationnistes ne peuvent constituer des coopératives autonomes. Ils doivent se contenter de défendre leurs arguments au sein d'unités dont le fonctionnement ne répond pas à leurs attentes. Ils cherchent à tirer parti des dysfonctionnements observés et se plaisent à souligner les difficultés rencontrées par leurs adversaires intégrationnistes.

Les intermédiaires intégrationnistes sont le plus souvent des jeunes ingénieurs agronomes originaires du village. Confinés dans des fonctions subalternes à la fin de la période communiste, ils entendent s'affirmer dans le nouveau régime. Pour adapter le programme défini par le SDS aux réalités locales, ils se glissent dans les nouvelles structures agraires. Leur démarche s'appuie sur le raisonnement suivant : les paysans sont attachés aux coopératives pour la raison qu'ils y ont vécu sans difficultés durant des décennies ; plutôt que de les en chasser par la force comme le SDS a initialement tenté de le faire en mettant sur pied les Comités de liquidation, il s'agit de les amener en douceur vers un autre type d'organisation. Il suffit d'imposer quelques principes intégrationnistes dans les coopératives pour amener les paysans à appréhender différemment leur avenir. Une fois gagnés par ce nouvel état d'esprit, les petits propriétaires adopteront automatiquement un comportement d'entrepreneur. Leur intérêt leur apparaîtra sous son vrai jour et les amènera à voter pour les candidats intégrationnistes. Dans cette optique, la coopérative n'est pas un modèle pérenne, mais seulement une " matrice d'apprentissage de conduites économiques autonomes $n 34$.

Les intermédiaires intégrationnistes peuvent éprouver quelques difficultés à s'imposer directement à la tête d'une coopérative. Il leur faut alors se glisser dans le conseil d'administration d'une unité constituée et tenter d'y imposer progressivement leurs vues. Lorsqu'un blocage est observé, la solution

${ }_{3}$ Cf. Creed (Gerald W.), " The Politics of Agriculture : Identity and Socialist Sentiment in Bulgaria ", Slavic Review, 54 (4), 1995.

34 Maurel (Marie-Claude), " La naissance de nouveaux acteurs sociaux sur la scène locale ", Revue d'Etudes Comparatives Est-Ouest, (4), 1994, p. 138. 
consiste à faire scission et à fonder une nouvelle coopérative avec les terres des paysans qui sont d'ores et déjà convertis aux thèses intégrationnistes.

Dans sa forme la plus commune, une coopérative intégrationniste est dirigée par un conseil d'administration restreint et ne compte aucun salarié. Les membres du conseil d'administration louent la terre des paysans et l'exploitent eux-mêmes. Lorsque la tâche est trop lourde - pendant les périodes de labour et de moisson par exemple - ils ont recours aux services d'une main d'œuvre extérieure qu'ils rémunèrent à la tâche. Les propriétaires sont rétribués sur une base forfaitaire : un revenu par hectare est arrêté au début de la saison pour chaque type de céréales. Les membres du conseil d'administration prennent à leur compte toutes les recettes et les dépenses de la coopérative ; leur revenu est constitué par les sommes qui restent dans les caisses de la coopérative une fois que les loyers ont été versés aux propriétaires 35 .

Les dirigeants des coopératives intégrationnistes ne se contentent pas de reconnaitre aux propriétaires le droit de recouvrer le plein usage de leur parcelle. Ils entendent également leur communiquer l'esprit d'initiative et le sens des responsabilités. Dans leur gestion courante, ils élèvent donc la flexibilité au rang de valeur cardinale : les paysans qui leur louent des terres sont encouragés à conserver une partie de leur domaine pour un usage individuel et à vendre librement la récolte qu'ils en tirent. Ils sont invités en d'autres termes à élaborer des stratégies mixtes. En apprenant à jongler avec plusieurs régimes de propriété, les petits exploitants agricoles doivent progressivement se défaire de la logique d'assistanat à laquelle le régime communiste les a accoutumé ${ }^{36}$.

Les intermédiaires intégrationnistes se réapproprient de la sorte les coopératives. Ils les détournent des objectifs conciliatoires qui ont présidé à leur édification et s'efforcent de les mettre au service du programme défendu par le SDS. Cette politique n'est pas appliquée sans encombre. Elle se heurte régulièrement à une double incompréhension.

Dans les premiers temps, l'appareil dirigeant du SDS peine à identifier ses intermédiaires : coupé des réalités locales et aveuglé par les combats qu'il livre contre l'ancienne nomenklatura au niveau national, il est tenu par la conviction que tous les dirigeants des coopératives indistinctement sont des potentats communistes en quête d'une nouvelle sinécure. Ayant travaillé dans les

35 Cf. Kaneff (Deema), " Private Cooperatives and Local Property Relations in Rural 8ulgaria ", Replika, (3). 1998 ; Yamal (Brent), "The land-use impacts of Bulgarian decollectivization ", in Paskaleva (Krassimira), Shapira (Philip), Pickles (John), Koulov (Boian), eds., Bulgaria in Transition, Aldershot : Ashgate, 1998.

$3^{6}$ Cf. Kaneff (Deema), "Responses to “Democratic" Land Reforms in Bulgaria " (art. cit.), p. 101 ; Swinnen (Johan F. M.), "On 1iquidation Councils, Flying troikas and Orsov Cooperatives: The Political Economy of Agricultural Reform in Bulgaria ", in Swinnen (Johan F. M.l, ed., Political Economy of Agrarian Reform in Central and Eastern Europe, Aldershot : Avebury, 1997. 
structures collectives sous le régime communiste, les ingénieurs agronomes sont regardés avec méfiance. Le fait qu'ils aient toujours été affectés à des postes secondaires n'est pas pris en considération. S'ils se revendiquent ouvertement du SDS, ils sont encore soupçonnés de se livrer à de basses manœuvre et de travailler secrètement pour le compte du BSP. Ils éprouvent donc les pires difficultés à obtenir les soutiens nationaux qu'ils réclament. Le parti ne leur accorde son investiture que "faute de mieux" et dans l'attente de voir émerger des figures locales plus assurement dévouées à sa cause.

Il arrive également que les paysans contrarient les desseins des intermédiaires intégrationnistes. Lorsque la récolte est abondante, les dirigeants de la coopérative réalisent des profits substantiels et les propriétaires reçoivent peu en comparaison. Une répartition plus équitable des bénéfices est alors réclamée. Les intérèts individuels s'expriment bien à l'intérieur de la coopérative, mais non sur le registre attendu ${ }^{37}$.

Les intermédiaires intégrationnistes sont mal compris par les dirigeants nationaux du SDS cornme par les paysans auxquels ils s'adressent. La démarche de leurs homologues conciliatoires prête moins à la méprise. Son succès n'est pas assuré pour autant.

Les intermédiaires conciliatoires sont le plus souvent des personnes âgées qui ont quitté le village pour des raisons professionnelles et qui s'y sont rétablies une fois venu l'âge de la retraite. Ils n'ont pas une expérience directe de l'agriculture, mais sont prêts à utiliser leurs économies pour fonder une coopérative. Selon leur point de vue, la compétition est nécessaire dans l'agriculture. Mais elle doit engager des coopératives plutôt que des individus motivés par des perspectives de gain à court terme. L'État a pour devoir de subventionner les coopératives déficitaires pour les remettre à flots et leur permettre de concourir à chances égales avec des unités plus rentables. Un écart doit être marqué avec l'anti-communisme outrancier du SDS : il s'agit de récuser la thèse selon laquelle le développement de l'agriculture bulgare passe par une éradication pure et simple des structures héritées du communisme ${ }^{38}$.

Les coopératives conciliatoires s'appuient dans une large mesure sur les terres de paysans qui, trop âgés ou contraints de résider en ville, ne peuvent se consacrer aux travaux des champs. Elles recrutent donc des employés à plein temps qu'elles rémunèrent au moyen de sommes prélevées sur le chiffre d'affaires. Les membres du conseil d'administration reçoivent eux-mêmes un salaire fixe. Les récoltes sont redistribuées aux paysans à proportion de la terre

37 Cf. Creed (Gerald W.), "The Politics of Agriculture " (art. cit.) ; Swinnen (Johan F. M.), art. cit. ; Iyons (Robert), Rausser (Gordon), Simon (Leo), " Disruption and continuity in Bulgaria's agrarian reform ", in Schmitz (Andrew) et al., Privatization of Agriculture in New Market Economies : Lessons from Bulgaria, Norwell : Kluwer Academic Publishers, 1994.

$3^{8}$ Cf. Creed (Gerald W.), "Deconstructing Socialism in Bulgaria " (art. cit.), p. 228. 
qu'ils ont engagée dans la coopérative. Les délimitations des parcelles ne font pas l'objet d'une attention scrupuleuse : la terre n'est pas conçue comme un bien privé mais comme une possession de la coopérative ; les membres de la coopérative se la représentent comme à une entité unifiée et non comme une agglomération de propriétés individuelles. Dans la gestion quotidienne, les intérêts de la coopérative priment les intérêts particuliers. Chaque année, un pourcentage prélevé sur les bénéfices vient alimenter un "fond général" et sert à subventionner l'école et la bibliothèque municipales 39 .

Les intermédiaires conciliatoires trouvent immédiaterment un soutien au niveau national. Le BSP valorise les coopératives qu'ils dirigent et les regarde comme les chevilles ouvrières de son programme agraire. Son jugement se fait plus sévère, toutefois, lorsque, soucieux d'entretenir leur popularité, les intermédiaires locaux font preuve de complaisance envers les paysans dont ils gèrent les terres. Pour ces derniers, les coopératives sont avant tout un moyen d'obtenir des produits de première nécessité et d'économiser à proportion l'argent obtenu par d'autres moyens. L'équilibre symbiotique établi sous le communisme entre travail des terres collectives et cultures de subsistance trouve dans cette posture un prolongement indirect. Pour contenter les paysans, les responsables des coopératives conciliatoires doivent donc revoir leurs objectifs de rendement à la baisse et renoncer à produire uniquement des céréales destinées aux circuits de consommation nationaux. Dans certains cas, ils vont jusqu'à doter la coopérative d'une unité de transformation qui achète elle-même une partie des céréales et des oléagineux pour revendre aux villageois du pain et de l'huile à bon marché4 ${ }^{\circ}$.

Un équilibre est recherché entre la fidélité au programme conciliatoire et la nécessité de complaire aux membres de la coopérative. Les intermédiaires locaux tentent de remontrer à leur appareil dirigeant qu'ils s'écartent de leurs préceptes conciliatoires pour mieux les défendre dans les faits et pour retenir les paysans de s'orienter vers des coopératives moins conformes aux desseins du BSP. Mais cet ordre de priorité local échappe le plus souvent aux responsables nationaux. Les relations entre la direction du parti et sa base se font de plus en plus tendues à mesure que les appréciations divergent. Si les intermédiaires conciliatoires bénéficiaient initialement d'un avantage sur leurs concurrents, ils finissent par se heurter à des difficultés de même ampleur.

Qu'ils défendent des options anti-intégrationnistes, intégrationnistes ou conciliatoires, les intermédiaires locaux bulgares doivent tous se livrer à un

39 Cf. les études de creed (Gerald W.) : : Between Economy and Ideology : Local Level Perspective on Political and Economic Reform in Bulgaria ", Socialism and Democracy, (13), 1991 ; "The Politics of Agriculture " (art. cit.) ; An Old Song in a New Voice " (art. cit.), p. 85.

40 Cf. Kaneff (Deema), "Responses to "Democratic" Land Reforms in Bulgaria " (art. cit.) ; Creed (Gerald W.), " Deconstructing Socialism in Bulgaria " (art. cit.). 
travail d'acclimatation : la grande majorité des paysans reste engagée dans des coopératives ; les militants installés dans les villages savent qu'il n'est pas possible de tourner le dos à cette réalité. Pour obtenir quelque succès électoral - et pour imposer le programme élaboré par leur parti - il leur faut se glisser dans les structures agraires existantes. Cette orientation peut générer quelques dissensions avec les appareils politiques nationaux. Elle amène dans tous les cas à nouer des relations ambivalentes avec les paysans. Les obstacles qu'elle rencontre de la sorte sont le fait de la logique de transvasement caractérisée plus haut. Lorsqu'une logique de drainage s'impose en lieu et place, les obstacles sont d'une autre nature ; l'emboîtement des logiques macro-institutionnelles et micro-institutionnelles ne produit pas les mêmes effets : les intermédiaires locaux se glissent dans les exploitations coopératives, mais ils les utilisent pour démontrer aux paysans la supériorité du programme qu'ils défendent.

\section{Un travail de démonstration}

En Roumanie, les intermédiaires locaux doivent composer avec une structure agraire bien différente de celle sur laquelle s'appuient leurs homologues bulgares. Les paysans ne sont pas engagés en grand nombre dans les sociétés agricoles. Les responsables des sociétés agricoles peuvent défendre des options anti-intégrationnistes, intégrationnistes, ou conciliatoires : ils doivent dans tous les cas mettre en lumière les avantages comparatifs du type d'organisation qu'ils privilégient. Cette entreprise ne peut toujours être menée à bien : par le fait des décalages observés entre les régimes de propriété, des stratégies individuelles sont élaborées qui interfèrent avec les objectifs fixés par les partis politiques.

Les intermédiaires anti-intégrationnistes sont issus de l'intelligentsia villageoise (médecins, enseignants, ...). Ils détenaient un pouvoir informel important dans la période communiste. Le changement de régime les indispose dans la mesure où il permet à des paysans peu qualifiés de s'enrichir et de s'élever au rang de notable tandis que leurs propres revenus stagnent et que leur prestige social s'effrite. Pour contrecarrer cette évolution, ils s'efforcent d'acquérir quelques revenus supplémentaires en se portant à la tête de sociétés agricoles ${ }^{4}$.

Dans les unités ainsi formées, les principes d'organisation restent proches de ceux qui étaient en vigueur dans les coopératives agricoles de production. L'objectif affiché est de cultiver les céréales les plus rentables sur un mode intensif. Une mécanisation maximale est recherchée. La répartition des cultures sur la surface totale est opérée sans que les délimitations des propriétés individuelles soient prises en considération. Les membres de la société agricole 
sont rémunérés en nature et en espèces à hauteur de $30 \%$ de la production totale. Les paysans âgés et les citadins qui confient leur parcelle à la société agricole sans participer aux tâches agricoles ne peuvent prétendre à plus de $10 \%$ des bénéfices ${ }^{4}$.

Si des paysans intègrent les sociétés agricoles anti-intégrationnistes et s'y maintiennent, les intermédiaires locaux ne peuvent en tirer immédiatement la conclusion que leur démarche est payante et que le programme défendu exerce un fort pouvoir d'attraction. Leur succès n'est dû, dans la majorité des cas, qu'à une absence d'alternative au niveau local : les paysans n'ont pas les moyens d'acquérir le matériel agricole qui leur permettrait de cultiver directement leur parcelle ; faute de pouvoir en louer à un propriétaire plus fortuné, ils doivent se résoudre à intégrer la société agricole constituée. Les dirigeants peuvent alors dicter leurs volontés en brandissant la menace d'une exclusion : un propriétaire dont la terre serait tenue à l'écart de la société agricole devrait renoncer à l'exploiter et à en tirer une forme quelconque de revenu43.

Les paysans n'adhèrent pas positivement au programme anti-intégrationniste. Dès l'instant qu'ils peuvent observer des sociétés agricoles constituées sur des bases différentes dans d'autres villages, ils se montrent insatisfaits de leur sort et refusent d'apporter leurs suffrages aux intermédiaires locaux antiintégrationnistes. Leur mécontentement se traduit par des comportements individualistes : lorsque les cultures de la société agricole sont affectées par des intempéries, les propriétaires dont les terres ont été épargnées refusent que les pertes soient assumées collectivement ; ils entendent que chacun soit rémunéré en considération des rendements de sa parcelle propre plutôt qu'en considération du rendement moyen de la société44. Pour étouffer ces revendications, les dirigeants doivent faire preuve de fermeté. Ils s'aliènent un peu plus encore les paysans. À défaut de compter sur des soutiens immédiats, ils s'attachent à faire la preuve que leur principe d'organisation donne les meilleurs résultats. Ils se persuadent que les tensions observées dans l'immédiat seront levées bientôt par cette démonstration d'efficacité.

Les efforts livrés par les intermédiaires intégrationnistes visent à convaincre les mêmes électeurs. Pour mettre en œuvre le programme défini par le PNTCD, il suffirait dans l'absolu de soutenir les dynamiques d'accumulation amorcées par les paysans les plus prospères en poussant les petits paysans à céder leurs terres : de grandes exploitations seraient ainsi constituées qui permettraient de pratiquer une agriculture intensive alignée sur les modè-

$4^{2}$ Cf. Kideckel (David A.), " Two Incidents on the Plains in Southem Transylvania " (art. cit.), pp. 57-60.

43 Cf. Verdery (Katherine), “Fuzzy Property : Rights, Power, and Identity in Transylvania's Decollectivization ", in Burawoy (Michael), Verdery (Katherine), eds., op. cit., p. 63.

44 Cf. Creed (Gerald W.), « Deconstructing Socialism in Bulgaria " (art. cit.). 
les occidentaux. Logique d'un point de vue économique, cette orientation serait néanmoins préjudiciable sur le plan électoral : les grands propriétaires ne formant que $5 \%$ de la paysannerie, ils ne sauraient permettre à un parti de s'implanter profondément dans les campagnes. La solution la plus indiquée consiste donc à diffuser l'esprit de compétition dans les masses rurales : il s'agit de convaincre les petits paysans d'adopter un comportement d'entrepreneur plutôt que de rester arc-boutés sur des cultures de subsistance. Si la nécessité d'une sélection entre les compétiteurs ne peut ètre dissimulée, l'idée doit s'imposer qu'un paysan consciencieux et travailleur verra toujours ses efforts récompensés et aura la possibilité d'amorcer à son tour une dynamique d'accumulation.

Dans la perspective ainsi tracée, les intermédiaires locaux doivent jouer un ròle d'exemple. Il leur faut remontrer à tous les villageois que l'esprit d'initiative permet à des propriétaires modestes de se transformer en grands paysans. Chacun doit pouvoir s'identifier eux et se convaincre qu'il peut suivre la même voie. Les intermédiaires désignés sont jeunes le plus souvent. Ils ont suivi une formation supérieure à la fin de la période communiste et ont exercé des fonctions d'encadrement dans les coopératives agricoles de production ou dans les entreprises agricoles d'État. Après le changement de régime, ils se sont convertis sans peine à " la nouvelle idéologie de l'entreprise privée "45. Pour servir leurs desseins, ils se portent à la tête de sociétés agricoles. Ils les utilisent ensuite comme des tremplins économiques : s'ils ne disposent pas individuellement d'une surface foncière et d'une capacité d'investissement suffisantes pour concurrencer les grands paysans, ils entendent s'affirmer par le truchement de structures coopératives. Ils ne s'engagent dans les sociétés agricoles qu'à titre transitoire et prévoient d'en sortir dès qu'ils disposeront d'un pactole suffisant pour amorcer seuls une dynamique d'accumulation ${ }^{46}$.

Ces stratégies contrarient parfois la logique de démonstration qu'elles sont supposées servir. À l'intérieur des sociétés agricoles, les intermèdiaires intégrationnistes mettent leur savoir et leurs relations au service de quelques manœuvres frauduleuses. S'ils parviennent à s'enrichir, c'est en combinant les avantages que leur procurent les structures coopératives et les activités qu'ils mènent sur des terrains annexes. Ils jouent de l'enchevêtrement des nouveaux statuts : attestation officielle à l'appui, ils déclarent apporter 2 ou 3 hectares de terres dans une société agricole ; une partie de cette superficie est, en fait, enclose dans une ferme d'État mitoyenne ; la même terre permet de cumuler indûment la part de la récolte versée par la société agricole et les dividendes dis-

45 Maurel (Marie-Claude), art. cit., p. 138.

46 Cf. Kideckel (David A.), "Populatia rurala si autoritatea în noua Românie " (art. cit.) ; Kideckel (David A.), op. cit., pp. 222-223 ; Hirschausen (Béatrice Von), op. cit., p. 322. 
tribués par la ferme d'État. Les intermédiaires intégrationnistes reversent aux petits paysans qui entrent dans leurs sociétés agricoles une quantité de céréales inférieure à celle que la taille de leur parcelle leur permettrait de réclamer ; ils vendent pour leur propre compte les surplus ponctionnés 47 . Ils s'enrichissent par des moyens détournés, au détriment des électeurs qu'ils ont pour tâche de convaincre : l'exemple qu'ils offrent dessert le modèle intégrationniste plutôt qu'il ne contribue à sa diffusion.

Les intermédiaires conciliatoires peuvent espérer tirer profit de ces difficultés. Plus âgés que leurs concurrents intégrationnistes, ils ont comme eux exercé des fonctions intermédiaires dans les coopératives agricoles de production et dans les entreprises agricoles d'État. Ils s'efforcent de construire des sociétés agricoles pérennes : il s'agit pour eux de proposer un modèle d'organisation qui conserve les avantages de la structure agraire instituée par le régime communiste sans renoncer aux perspectives offertes par l'économie de marché. Dans les unités de production qu'ils mettent sur pied, tous les bénéfices sont redistribués aux propriétaires en considération de la quantité de terre fournie. Les paysans qui engagent leur parcelle dans la société agricole ont l'obligation de participer aux travaux des champs ; aucune place n'est faite aux paysans âgés et aux propriétaires qui ne peuvent résider sur place ${ }^{4}$.

Les sociétés agricoles constituées sur ce mode sont favorisées : une parfaite cohérence peut être relevée entre leur mode de fonctionnement et le programme agraire qu'elles visent à conforter. Les intermédiaires locaux qui les dirigent n'en connaissent pas moins quelques difficultés. Les membres d'une société agricole sont libres de recouvrer à tout moment le plein usage de leur parcelle. Ils tirent aisément parti de cette latitude : ils restent engagés dans les structures coopératives jusqu'à ce que leur terre ait été labourée puis s'en retirent pour jouir seuls de leur récolte. Afin de lutter contre ces pratiques, les dirigeants des sociétés agricoles sont parfois tentés de faire signer un contrat pluriannuel aux paysans qui leur confient une terre. Des protestations se font aussitôt entendre et le risque se profile de voir les propriétaires s'orienter vers des formes d'organisation concurrentes. Le seul moyen de les retenir est alors de leur offrir une rémunération élevée en limitant au strict minimum la part des bénéfices consacrée aux investissements : des semences et des engrais sont achetés pour la saison suivante mais aucun matériel supplémentaire n'est acquis par la société agricole. Si cette option offre des avantages immédiats, elle compromet le développement des activités agricoles dans le

47 Cf. Verdery (Katherine), What Was Socialism and What Comes Next (op. cit.), pp. 141-143, 162 ; Verdery (Katherine), "Disambiguating ownership : Rights and power in Transylvania's decollectivation " (art. cit.) ; Verdery (Katherine), "Fuzzy Property : Rights, Power, and Identity in Transylvania's Decollectivization " (art. cit.) ; Sandu (Dimitru), " Cine sunt antreprenorii din agricultura de tranzitie ? ", Sociologie Româneasca, (1), 1999, pp. 34-36 ; Sandu (Dimitru), " Drumul antreprenorial : fara încredere dar cu relatii ", Socilogie Româneasca, (2), 1999, pp.126-128.

$4^{8}$ Kideckel (David A.), " Two Incidents on the Plains in Southern Transylvania " (art. cit.), pp. 57-60. 
moyen terme et interdit d'approcher l'objectif de productivité fixé par le programme conciliatoire. Soucieux de mener leur mission à bien, des intermédiaires locaux tentent parfois de contourner les menaces de retrait formulées par les paysans ; ils s'attachent à démontrer que des investissements réalisés en commun sont une promesse de gains futurs. Dans le cas de figure même où les propriétaires sont sensibles à ce discours, le risque d'instrumentalisation n'est pas écarté : un paysan peut choisir d'engager quelques hectares de terrain dans la société agricole et de conserver le libre usage de la surface restante ; si réduite soit-elle, sa participation à la société lui donne un titre de propriété sur le matériel acheté et lui confère le droit de l'utiliser à son gré, y compris sur les terres qu'il a maintenues à l'écart des structures coopératives ${ }^{49}$. La combinaison de plusieurs régimes de propriété permet d'élaborer des stratégies particulières qui interfèrent avec les projets de développement mis au point par les intermédiaires conciliatoires.

Pour ne pas cumuler l'impopularité et l'inefficacité, les dirigeants des sociétés agricoles optent le plus souvent pour une logique de court terme et se résolvent à redistribuer la quasi-totalité des bénéfices. Cette politique permet d'obtenir un effet de démonstration immédiat mais elle expose ses promoteurs à quelques retours de bâton : si une société agricole conciliatoire peine à se développer faute d'investissements, elle risque de se trouver en plus mauvaise posture que les unités de production qui servent à diffuser un programme concurrent.

En Roumanie comme en Bulgarie des obstacles se dressent sur le chemin que tentent de tracer les intermédiaires locaux. Ces obstacles présentent des formes différentes selon que les mesures de décollectivisation ont été guidées par telle ou telle logique, mais ils sont toujours conséquents. Les intermédiaires conciliatoires bénéficient, au départ, d'un net avantage sur leurs concurrents anti-intégrationnistes et intégrationnistes : les coopératives sont parfaitement accordées avec le programme agraire qu'ils sont chargés de diffuser auprès des paysans ; un emboitement optimal peut être observé entre les structures macro-institutionnelles et la démarche qu'ils adoptent au niveau micro-institutionnel. L'équilibre se révèle pourtant précaire : la prise en compte de quelques impératifs locaux amène rapidement à marquer un écart avec les objectifs affichés à l'échelle nationale. Cette tendance générale laisse cours à des trajectoires diversifiées. En Bulgarie, les intermédiaires conciliatoires se livrent dans les premiers temps à un travail d'acclimatation efficace. À la différence de leurs homologues anti-intégrationnistes, ils peuvent compter sur une adhésion positive des paysans à leur démarche. Par contraste avec leurs adversaires intégrationnistes, ils sont soutenus sans réserve par la direction de leur parti. Soucieux de ne pas mécontenter les membres de la

49 Cf. Verdery (Katherine), "Fuzzy Property : Rights, Power, and Identity in Transylvania's Decollectivization " (art. cit.), pp. 61,77.

En 1995 et 1996, 81,8\% des paysans roumains interrogés déclarent ne posséder en propre aucune machine agricole (Nemenyi (Agnes), art. cit., p. 73). 
coopérative, ils en viennent pourtant à adopter des mesures qui paraissent contrarier les objectifs définis au niveau national. En Roumanie, les intermédiaires conciliatoires peuvent démontrer que leurs coopératives sont plus efficaces que les concurrentes dans la mesure où elles s'inscrivent dans le prolongement de la politique agraire mise en oeuvre au lendemain de la décollectivisation. Pour retenir les paysans d'expérimenter d'autres formules, il leur faut toutefois dispenser des avantages immédiats et limiter les dépenses d'investissement. La démonstration menace de tourner court dans le moyen terme, sans que les relations avec la direction du parti soient aucunement en cause.

\section{CONCLUSION}

Pour comprendre les fortunes diverses que rencontrent les partis politiques bulgares et roumains dans leurs tentatives d'implantation locale, il est nécessaire d'écarter à la fois les thèses de la désagrégation et de l'imprégnation. Il s'agit de mettre en évidence un "fondu enchaîné" entre le régime communiste et la période présente, tant au niveau macro-institutionnel qu'au niveau micro-institutionnel. Quatre plans successifs se relaient ainsi dans une parfaite solution de continuité. La relation au pouvoir communiste doit être envisagée en première instance : les cultures individuelles et le secteur socialiste sont restés parfaitement intégrés en Bulgarie tandis qu'ils se sont contrariés en Roumanie ; les relations agraires ont été harmonieuses dans le premier cas et tendues dans le second. Il convient ensuite d'envisager la réaction que la paysannerie oppose au changement de régime : les paysans bulgares se maintiennent dans des structures coopératives tandis que leurs voisins roumains s'empressent de jeter bas le système agraire édifié par les autorités communistes. Il est alors possible de comprendre le fonctionnement des nouveaux modèles d'organisation introduits dans chaque cas de figure : en Bulgarie, les coopératives demeurent sur pied et leur statut est simplement réformé - ce qui permet à la majorité des paysans d'y rester engagés ; en Roumanie, des sociétés agricoles sont formées qui visent à regrouper les terres de petits paysans pour lors repliés sur des cultures de subsistance. La lumière peut enfin être faite sur le rôle joué par les intermédiaires locaux des partis politiques : dans le cas bulgare, les coopératives sont utilisées pour amener les paysans qui y sont d'ores et déjà engagés à adopter des comportements conformes à tel ou tel programme ; dans le cas roumain, les sociétés agricoles servent à montrer aux petits propriétaires repliés sur leur parcelle que des nouvelles perspectives peuvent s'ouvrir à eux s'ils adhèrent à tel programme plutôt qu'à tel autre.

Les paysans ne forment pas une masse ductile que les partis politiques pourraient manipuler à leur guise. Ils ne votent pas comme un seul homme 
pour le responsable de la coopérative ou de la société agricole dans laquelle ils sont engagés. Ils réagissent bien plutôt aux propositions que leur adressent les intermédiaires locaux. Ils les discutent, les comparent et les reformulent au besoin. Le programme conciliatoire s'attire leurs faveurs dans un premier temps pour la raison qu'il permet d'emboîter les structures agraires formées au niveau macro-institutionnel et la démarche adoptée par les intermédiaires locaux au niveau micro-institutionnel. Encore cet emboîtement ne peut-il être maintenu dans la durée. Lorsque les intermédiaires locaux doivent se livrer à un travail d'acclimatation, ils se plient aux exigences du terrain et entretiennent des relations difficiles avec la direction de leur parti. Lorsqu'ils s'essaient à un travail de démonstration, ils doivent satisfaire les demandes immédiates des paysans au risque de compromettre la bonne marche de la coopérative. Dès le moment que l'option conciliatoire perd en efficacité, une grande liberté de choix est offerte aux paysans : aucun programme ne pouvant écraser les autres, chacun peut décider d'en privilégier un puis de s'orienter vers un autre. Toute médaille ayant son revers, cette ouverture peut également générer une forme de découragement : puisqu'aucun modèle ne fait la preuve de son efficacité, les paysans perdent espoir et jettent le même opprobre sur tous les partis politiques indistinctement ${ }^{50}$. Cette posture peut expliquer les succès récents de quelques partis atypiques. Constitué quelques mois seulement avant les élections législatives organisées en Bulgarie le 19 juin 2001 - et incapable par conséquent de s'appuyer sur des intermédiaires locaux - le Mouvement national Siméon II (Nacionalno Dvišenie Simeon Tvori, NDST) est parvenu à recueillir 43,04 \% des voix. Alors qu'il n'a jamais réussi a s'implanter dans les municipalités rurales, le Parti de la Grande Roumanie (Partidul România Mare, PRM) a attiré $19,48 \%$ et $21,01 \%$ des suffrages aux élections législatives et sénatoriales du 26 novembre 2000 ; son président, Corneliu Vadim Tudor, a recueilli $28,34 \%$ des suffrages au premier tour de l'élection présidentielle organisée à la même date, résultat qui lui a permis de se présenter au second tour contre Ion Iliescu. Dans les deux cas, l'abstentionnisme des paysans a permis à des formations principalement soutenues par les populations urbaines d'obtenir des scores sans précédent.

De nouvelles recherches sont nécessaires pour apprécier les effets de cette évolution : il s'agit d'évaluer la capacité de réaction des partis traditionnels et de déterminer dans quelle mesure les problèmes observés à la base peuvent influer sur les programmes élaborés au niveau national.

50 Cf. Ivanova (Radost), "Social change as reflected in the lives of Bulgarian Villagers n, in Kideckel (David A.), ed., op. cit., pp. 234-235 ; Vultur (Mircea), " Marginalité et exclusion politique de la paysannerie roumaine ", La Nouvelle Alternative, (48), 1997. 